\title{
Effect of Trichlormethiazide and Captopril on Nitric Oxide Synthase Activity in the Kidney of Deoxycorticosterone Acetate-salt Hypertensive Rats
}

\author{
Atsuko TaKanohashi, MD, Akihiro Tojo, ${ }^{*} \mathrm{MD}$, \\ Naohiko Kobayashi, MD, Shigeru YAGI, MD, \\ and Hiroaki MATSUOKA, MD
}

\section{SUMMARY}

Nitric oxide (NO) production is reduced in patients with essential hypertension and in some experimental models. We have investigated the effect of trichlormethiazide and captopril on NO synthase (NOS) activity and glomerular damage in the kidney of deoxycorticosterone acetate (DOCA)-salt hypertensive rats. DOCA-salt rats were induced with weekly injections of DOCA (30 mg/kg body weight $(\mathrm{BW})$ ) and $1 \%$ saline in drinking water after right nephrcctomy. As antihypertensive thcrapies, CAP (captopril, $40 \mathrm{mg} / \mathrm{kg}$ BW) and TCM (trichlormethiazide, $10 \mathrm{mg} / \mathrm{kg} \mathrm{BW}$ ) were given after induction of DOGA-salt hypertension. The increased blood pressure was significantly lowered by TCM, but not by CAP after 5 weeks. Nitrite production in kidney slices was suppressed in DOCA-salt rats, and immunoreactivity for both braintype NOS (B-NOS) in macula densa and endothelial-type NOS (EG-NOS) in renal vessels was decreased. TCM significantly increased the nitrite production in the kidney slices and B-NOS immunoreactivity, whereas these changes were less in CAP. Glomerulosclerosis score was significantly higher in DOCA-salt rats, and TCM ameliorated renal damage more effectively than CAP. These results indicate that the reduced nitrite production in the kidney of DOCA-salt hypertensive rats was increased more effectively by trichlormethiazide than by captopril, via increased immunoreactivity for B-NOS in the macula densa, and prevented renal damage. (Jpn Heart J 1996; 37; 251-259)

Key words: Nitric oxide synthase (NOS) Nitrite Hypertension Antihypertensive therapy Macula densa

T $\mathrm{T}$ has been shown that nitric oxide production is reduced in patients with essential hypertension. ${ }^{1{ }^{2 !}}$ In some animal models of hypertension such as the

\footnotetext{
From the Division of Hypertension and Cardiorenal Diseases, Department of Internal Medicine, Dokkyo University School of Medicine, Mibu, Tochigi, Japan

* Present address and address for correspondence: Akihiro Tojo, MD, The Second Department of Internal Medicine, Faculty of Medicine, University of Tokyo 7-3-1 Hongo, Bunkyo-ku, Tokyo 113, Japan.

Received for publication October 9, 1995.

Accepted November 27, 1995.
} 
Dahl salt sensitive rat $^{3,4)}$ and deoxycorticosterone acetate (DOCA)-salt hypertensive rat, ${ }^{5,6)}$ NO production is also decreased. Since normalization of blood pressure by antihypertensive therapies improved the decreased endothelium-dependent relaxation, it is thought that a reduction in NO production is a result of endothelial damage secondary to hypertension ${ }^{7}$. However, it is not known whether antihypertensive drugs have a direct effect on NO synthase (NOS) activity, and whether this effect is different among various antihypertensive drugs.

In the kidney, both constitutive and inducible NOS isoforms have been shown to exist in normal rats. ${ }^{8-13)}$ Rats treated with a high salt diet demonstrated a decrease in immunoreactivity of brain-type constitutive NOS (B-NOS) in the macula densa, and vascular smooth muscle cell-type inducible NOS (VSM-NOS) in terminal afferent arterioles. ${ }^{14)}$ Since DOCA-salt hypertension is a circulation volume-dependent type of hypertension with a high salt intake, immunoreactivity for B-NOS might be suppressed in the macula densa, and trichlormethiazide, a diuretic drug may induce some change in B-NOS activity. Angiotensin converting enzyme inhibitor has been shown to stimulate formation of NO in the endothelial cells of the skin microvasculature. ${ }^{15)}$ The $\mathrm{AT}_{1}$ receptor antagonist, losartan has been shown to increase the immunoreactivity of B-NOS and VSM-NOS in the kidney. ${ }^{(4)}$ Thus, the salt concentration in the macula densa and renin-angiotensin system may independently regulate NOS activity in the kidney.

In the present study, we have investigated the effects of trichlormethiazide and captopril on NOS activity and glomerular damage in DOCA-salt hypertensive rat kidney.

\section{Methods}

Animals and experimental designs: Thirty-four male Wistar rats weighing 250-300 g were used, and DOCA-salt hypertension was induced in twenty-six rats as described previously. ${ }^{16)}$ Briefly, rats received weekly subcutaneous injections of DOCA ( $30 \mathrm{mg} / \mathrm{kg}$ BW) after right nephrectomy and were given $1 \%$ saline for drinking water (DOCA-salt rats). Two groups of DOCA-salt rats were treated with different antihypertensive drugs mixed with normal rat pellets; 9 DOCA-salt rats were given $40 \mathrm{mg} / \mathrm{kg}$ BW/day captopril (CAP group) and 8 DOCA-salt rat $10 \mathrm{mg} / \mathrm{kg}$ BW/day trichlormethiazide (TCM group). The remaining 9 DOCA-salt rats were given normal rats pellets (DOCA group). Eight normal rats fed only rats pellets and tap water served as controls. Blood pressure was measured by the tail-cuff method, and 24 hour urinary protein excretion was measured using individual metabolic cages after 2 and 5 weeks. After 5 weeks of treatment, the kidneys were perfused with phosphate buffer followed by perfusion 
with periodate-lysine-paraformaldehyde (PLP) solution. The tissue was used for assay of nitrite production and histological examination.

Nitrite production in kidney slices: Three $50 \mu \mathrm{m}$ sections of each kidney were cut using a vibratome and incubated with $10 \mathrm{mM} \mathrm{L}$-arginine (L-Arg) in a buffer ( $\mathrm{pH}$ 7.2) containing $25 \mathrm{mM} \mathrm{N}$-2-hydroxyethylpiperazine-N'-2ethanesulfonic acid (HEPES) (Sigma, St. Louis, MO, USA), $140 \mathrm{mM} \mathrm{NaCl}, 5.4$

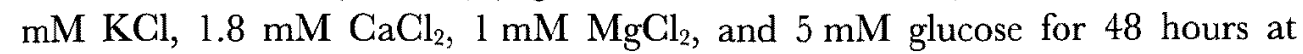
$37^{\circ} \mathrm{C}$. The supernatant was used for the assay of $\mathrm{NO}_{2}^{-}$production. The $100 \mu \mathrm{l}$ samples were incubated with $0.3 \mathrm{~N} \mathrm{NaOH}$ and $5 \% \mathrm{ZnSO} 4$, and centrifuged to remove proteins. Nitrite was measured by the Griess method with a $540 \mathrm{~nm}$ wavelength using an autoanalyzer (TCI-NOX 1000 m; Tokyo Kasei Kogyo, Tokyo, Japan). The amount of nitrite production was corrected for the protein level measured by the Bradford method (Bio-Rad, Richmond, CA, USA). In order to establish an optimal incubation time, nitrite production was measured after incubation for 4, 12, 24 and 48 hours using kidney slices from control animals $(n=6)$. Some slices were incubated with $10 \mathrm{mM} \mathrm{N} \mathrm{N}^{\mathrm{G}}$-monomethyl-L-arginine (L-NMMA) (Wako, Osaka, Japan) for 48 hours in order to ensure that nitric oxide was produced in the kidney slices.

\section{Immunohistochemistry of NO synthase and evaluation of glomerular dam-}

age: Two $\mu \mathrm{m}$ wax sections were processed for the streptavidin-biotin-horseradish peroxidase complex technique as mentioned previously. ${ }^{14)}$ Briefly, sections were dewaxed and incubated with $3 \% \mathrm{H}_{2} \mathrm{O}_{2}$ to eliminate endogenous peroxidase activity. The sections were incubated with the monoclonal antibodies against BNOS or EG-NOS (Transduction Laboratories, Lexington, KY, USA) with a 1:100 dilution for $60 \mathrm{~min}$ after being exposed to blocking serum. The sections were rinsed with phosphate buffered saline (PBS), and biotinylated secondary antibody against mouse immunoglobulin (Dako, Denmark) was applied for 20 $\min$. After being rinsed with PBS, the sections were incubated with the horseradish peroxidase (HRP)-conjugated streptavidin for $20 \mathrm{~min}$. HRP labeling was detected by the peroxidase substrate solution diaminobenzidine. The sections were then counterstained with hematoxylin and examined under a light microscope.

The other sections were also stained with periodic acid Schiff, and evaluated for glomerular sclerosis using a scoring system as follows; S0: normal glomerulus, S1: mild sclerosis (less than 25\%), S2: moderate segmental sclerosis (26$50 \%)$, S3: severe sclerosis $(51-75 \%)$, S4: global sclerosis $(76-100 \%)$. The glomerulosclerosis score is defined as $(0 \times$ number of $\mathrm{S} 0+1 \times$ number of $\mathrm{S} 1$ $+2 \times$ number of $\mathrm{S} 2+3 \times$ number of $\mathrm{S} 3+4 \times$ number of $\mathrm{S} 4$ ) $/$ (number of $\mathrm{S} 0+\mathrm{S} 1+\mathrm{S} 2+\mathrm{S} 3+\mathrm{S} 4)$.

Statistical analysis: The data are expressed as mean \pm SEM. The differences among the groups were analyzed based on a one-way analysis of variance. Differ- 
ences were considered to be significant at a $\mathrm{p}$ level below 0.05 .

\section{Results}

Effects of antihypertensive therapies on blood pressure and urinary protein excretion: The blood pressure of DOCA rats was significantly higher than the control rats (132 \pm 6 in the controls vs. $158 \pm 4 \mathrm{mmHg}$ in DOCA, $p<0.01$ ), and two treated groups showed significantly lower blood pressure than the DOCA group at the 2nd week (143 \pm 4 in CAP and $143 \pm 3 \mathrm{mmHg}$ in TCM, both $p<0.05$ vs. DOCA). At the 5 th week, the blood pressure of the DOCA group increased further $(129 \pm 5$ vs. $172 \pm 8, p<0.001)$. TCM therapy was effective in lowering the blood pressure significantly $(143 \pm 4, p<0.05)$, but CAP could not decrease the blood pressure (168 $\pm 4, \mathrm{NS}$ vs. DOCA) (Figure 1). The urinary protein excretion increased significantly in the DOCA group after 5 weeks $(15 \pm$ 3 vs. $218 \pm 62 \mathrm{mg} /$ day, $p<0.05)$. The CAP group did not show a reduction in proteinuria compared to the DOCA group $(287 \pm 65, \mathrm{NS})$, whereas the TCM group showed slightly lower urinary protein excretion $(125 \pm 55, \mathrm{NS})$.

Effect of antihypertensive therapies on nitrite production in kidney slices: Kidney slices from normal rats produced nitrite in a time-dependent manner $(4.26 \pm 0.14,5.64 \pm 0.23$, and $6.16 \pm 0.37 \mathrm{nmol} / \mathrm{mg}$ protein, 12,24 and 48 hours), and L-NMMA significantly inhibited nitrite production at 48 hours (4.85

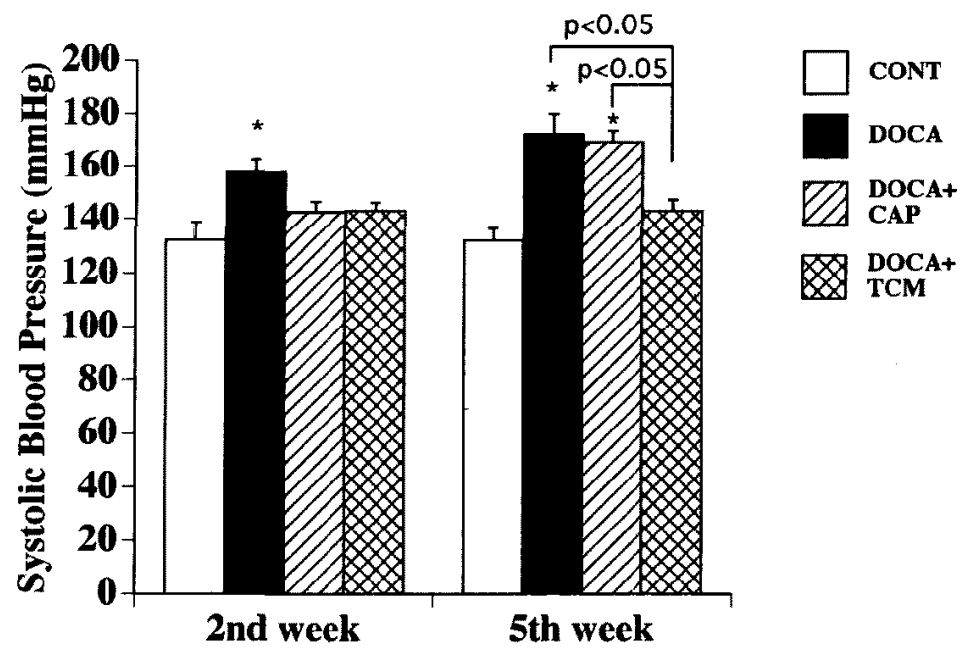

Figure 1. Effect of antihypertensive therapies on systolic blood pressure. Blood pressure was significantly increased in DOCA-salt rats after 2 weeks. Blood pressure was significantly lowered by trichlormethiazide (TCM) therapy after 5 weeks, but not by captopril (CAP) therapy. ${ }^{*} p<0.01$ vs. control. 


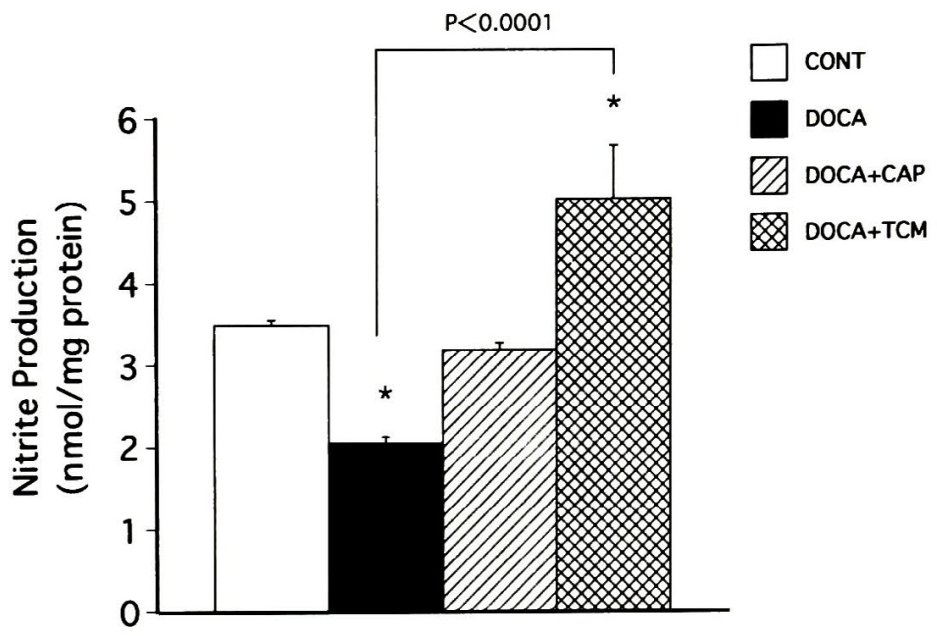

Figure 2. Effect of antihypertensive therapies on nitrite production in kidney slices. Nitrite production was suppressed in DOCA-salt rats. It was significantly increased by TCM therapy, but only slightly by CAP therapy. ${ }^{*} p<0.05$ vs. control.

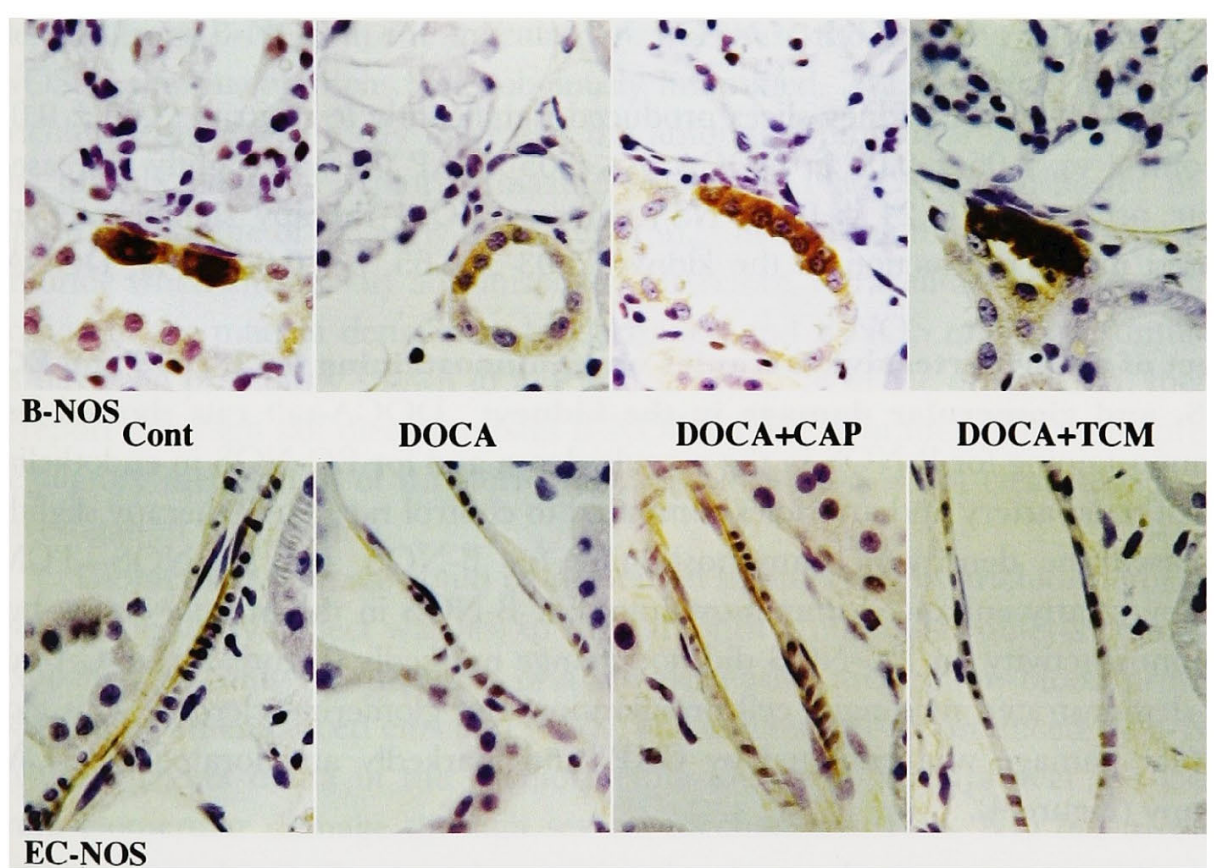

Figure 3. Effect of antihypertensive therapies on immunoreactivity for B-NOS and EC-NOS. Immunoreactivity for B-NOS in macula densa and EC-NOS in afferent arterioles was less in DOCA-salt rats than in the control rats. CAP therapy increased immunoreactivity for both B-NOS and EC-NOS, and TCM therapy markedly increased B-NOS staining in macula densa, but not EG-NOS. 


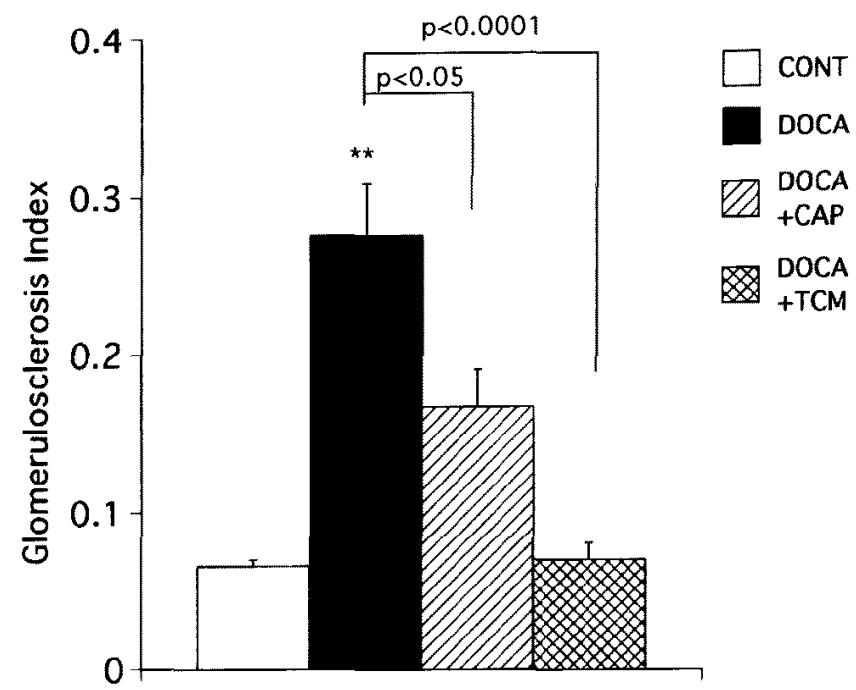

Figure 4. Effect of antihypertensive therapies on glomerulosclerosis score. Glomerular sclerosis index was significantly higher in DOCA-salt rats compared to control rats. This score was significantly lowered by CAP therapy, and normalized by TCM therapy. $* *_{p}<0.001$ vs. control.

$\pm 0.14, p<0.05)$.

DOCA-salt rat kidney slices produced significantly less nitrite $(3.50 \pm 0.07$ in control vs. $2.06 \pm 0.08$ in DOCA, $p<0.05)$. CAP therapy slightly increased nitrite production $(3.24 \pm 0.09, \mathrm{NS})$, whereas TCM therapy significantly increased nitrite production in the kidney $(5.03 \pm 0.65, p<0.0001$, vs. DOCA) (Figure 2).

Effect of antihypertensive therapies on immunostaining for B-NOS and EGNOS, and glomerular damage in the kidney: DOCA-salt rats showed less immunostaining for B-NOS in the macula densa and for EC-NOS in endothelial cells of renal artery and arterioles compared to control rats. CAP therapy slightly increased the density of immunostaining for B-NOS and EC-NOS. TCM therapy clearly enhanced immunostaining for B-NOS in the macula densa, but immunoreactivity for EG-NOS did not change markedly (Figure 3). DOCA-salt rats demonstrated mesangial cell proliferation and glomerulosclerosis. This glomerular damage was inhibited by CAP, and markedly ameliorated by TCM therapy (Figure 4).

\section{Discussion}

DOCA-salt hypertensive rat kidney slices demonstrated reduced NO production, and the immunoreactivity for both B-NOS in the macula densa and EC- 
NOS in endothelial cells in the kidney was also decreased. These results are consistent with previous reports which found that NO release and vasodilatation response to acetylcholine were decreased in the isolated perfused kidney of DOCA-salt hypertensive rats. ${ }^{5,6)}$ The mechanism of reduced NO production in hypertension is thought to be the result of hypertensive endothclial damage, ${ }^{1,2)}$ and the normalization of blood pressure ameliorated NO production. ${ }^{7)}$ However, in the present study, we have demonstratcd a reduction in immunoreactivity for B-NOS in the macula densa which could not be explained by mechanical damage caused by high blood pressure. Thus, high salt diet or deoxycorticosterone could have a direct inhibitory effect on B-NOS or EC-NOS activity.

As DOCA-salt hypertension is volume dependent, diuretics such as trichlormethiazide lowered blood pressure more effectively than the angiotensin converting enzyme inhibitor captopril. Nitrite production was increased with both antihypertensive therapies, which is partially explained by the protection of the endothelium from hypertensive damage. Actually, EC-NOS immunoreactivity was slightly restored by antihypertensive therapies, especially by captopril. However, nitrite production was increased more in the trichlormethiazide group than in the captopril group or control. This increased nitrite might be mainly derived from B-NOS in the macula densa because the immunoreactivity for BNOS in the macula densa was obviously intensified. These results indicate that trichlormethiazide directly increased the amount of immunoreactive B-NOS in the macula densa. Trichlormethiazide blocks the $\mathrm{Na}^{+}-\mathrm{Cl}^{-}$cotransporter in the apical membrane of the early distal nephron, ${ }^{177}$ decreasing total body sodium and chloride when chronically administered. Therefore, intracellular sodium concentration in the macula densa may be decreased and B-NOS might be stimulated as has been previously shown in low salt diet rats. ${ }^{14)}$ On the other hand, there is a report that high salt diet stimulates B-NOS activity in brain. ${ }^{18)}$ Thus, the precise regulatory mechanism of trichlormethiazide on B-NOS is not clear, and further investigation is necessary.

Glomerular damage could be prevented significantly by trichlormethiazide, but this protective effect was less in the captopril group. This difference may be explained not only by the effect of antihypertensive therapy on blood pressure, but also by an enhanced effect on NOS. The enhanced NO produced by B-NOS in the macula densa in the trichlormethiazide group may protect the kidney from glomerular damage through several mechanisms. First, NO has a role in tubuloglomerular feedback and maintains the glomerular filtration rate. ${ }^{9)}$ Second, NO inhibits mesangial cell proliferation. ${ }^{19}$ Third, it inhibits intraglomerular coagulation and prevents the release of various growth factors from platelets. ${ }^{20}$ Finally, $\mathrm{NO}$ inhibits $\mathrm{Na}^{+}-\mathrm{K}^{+}$-ATPase in renal tubules, increasing $\mathrm{Na}^{+}$excretion ${ }^{21)}$ and lowering blood pressure more effectively. Through these effects, 
trichlormethiazide, which increases B-NOS in the macula densa, is more effective in preventing renal damage in DOCA-salt hypertensive rats. Captopril also showed a weaker but significant effect in the prevention of glomerulosclerosis, even though captopril did not lower blood pressure significantly. The effect of angiotensin converting enzyme (ACE) inhibitors such as captopril in the prevention of glomerular damage is considered to be due to efferent arteriolar dilatation and a reduction in intraglomerular pressure. ${ }^{22}$ Moreover, it has been demonstrated that the beneficial effects of ACE inhibitors on renal injury were due to an increase in vasodepressor eicosanoids and $\mathrm{NO}$ production as well as inhibition of oxygen radical generation in injured renal tissue. ${ }^{23)}$ In our experiments, captopril might prevent glomerulosclerosis via these multiple factors even though NO production in kidney was not significantly increased.

In conclusion, nitrite production was reduced in DOCA-salt hypertensive rat kidney slices. Trichlormethiazide increased nitrite production in the kidney more effectively than captopril, via increased B-NOS in the macula densa, and prevented renal damage.

\section{REFERENCES}

1. Panza JA, Casino PR, Kilcoyne CM, et al. Role of endothelium-derived nitric oxide in the abnormal endothelium-dependent vascular relaxation of patients with essential hypertension. Circulation 1993; 87: 1468-74.

2. Panza JA, Quyyumi AA, Gallahan TS, et al. Effect of antihypertensive treatment on endotheliumdependent vascular relaxation in patients with essential hypertension. J Am Coll Cardiol 1993; 21 : II $45-51$.

3. Boegehold MA. Reduced influence of nitric oxide on arteriolar tone in hypertensive Dahl rats. Hypertension 1992; 19: 290-5.

4. Chen PY, Sanders PW. L-arginine abrogates salt-sensitive hypertension in Dahl/Rapp rats. J Clin Invest 1991; 88: 1559-67.

5. Hayakawa H, Hirata Y, Suzuki E, et al. Mechanisms for altered endothelium-dependent vasorelaxation in isolated kidneys from experimental hypertensive rats. Am J Physiol 1993; 264: H1535 41.

6. Hirata Y, Hayakawa H, Suzuki E, et al. Direct measurements of endothelium-derived nitric oxide release by stimulation of endothelin receptors in rat kidney and its alteration in salt-induced hypertension. Circulation 1995; 91: 122935.

7. Luscher TF, Vanhoutte PM, Raij L. Antihypertensive treatment normalizes decreased endotheliumdependent relaxation in rats with salt-induced hypertension. Hypertension 1987; 9 (Suppl III): III-1937.

8. Mundel P, Bachmann S, Bader M, et al. Expression of nitric oxide synthase in kidney macula densa cells. Kidney Int 1992; 42: 1017-9.

9. Wilcox CS, Welch WJ, Murad F, et al. Nitric oxide synthase in macula densa regulates glomerular capillary pressure. Proc Natl Acad Sci USA 1992; 89: 11993-7.

10. Tojo A, Gross SS, Zhang L, et al. Immunocytochemical localization of distinct isoforms of nitric oxide synthase in the juxtaglomerular apparatus of normal rat kidney. J Am Soc Nephrol 1994; 4: 1438-47.

11. Ujiie $K$, Yuen J, Hogarth L, et al. Localization and function of endothelial NO synthase $\mathrm{mRNA}$ expression in rat kidney. Am J Physiol 1994; 267: F296-302.

12. Tojo A, Guzman NJ, Garg LC, et al. Nitric oxide inhibits bafilomycin-sensitive $\mathrm{H}^{+}$-ATPase activity in 
rat cortical collecting duct. Am J Physiol 1994; 267: F509-15.

13. Ann KY, Mohaupt MG, Madsen KM, et al. In situ hybridization localization of mRNA encoding inducible nitric oxide synthase in rat kidney. Am J Physiol 1994; 267: F748-57.

14. Tojo A, Madsen KM, Wilcox CS. Expression of immunoreactive nitric oxide synthase isoforms in rat kidney. Effects of dietary salt and losartan. Jpn Heart J 1995; 36: 389-98

15. Warren JB, Loi RK. Captopril increases skin microvascular blood flow secondary to bradykinin, nitric oxide, and prostaglandins. FASEB J 1995; 9: 411-18.

16. Tojo A, Kimura $\mathrm{K}$, Nanba $\mathrm{S}$, et al. Variations in renal arteriolar diameter in deoxycorticosterone acetate-salt hypertensive rats; a microvascular study. Virchows Arch A 1990; 417: 389-93.

17. Ellison DH, Velazquez H, Wright FS. Thiazide-sensitive sodium chloride cotransport in early distal tubule. Am J Physiol 1987; 253: F546-54.

18. Villar MJ, Ceccatelli S, Ronnqvist M, et al. Nitric oxide synthase increases in hypothalamic magnocellular neurons after salt loading in the rat. An immunohistochemical and in situ hybridization study. Brain Res 1994; 644: 273-81.

19. Shultz PJ, Tayen MA, Marletta MA, et al. Synthesis and action of nitric oxide in rat glomerular mesangial cells. Am J Physiol 1991; 261: F600-6.

20. Shultz PJ, Raij L. Endogenously synthesized nitric oxide prevents endotoxin-induced glomerular thrombosis. J Clin Invest 1992, 90: 1718-25.

21. Mattson DL, Roman RJ, Cowley AW. Role of nitric oxide in renal papillary blood flow and sodium excretion. Hypertension 1992; 19: 766-9.

22. Anderson S, Brenner BM. Therapeutic implications of converting-enzyme inhibitors in renal disease. Am J Kidney Dis 1987; 10 (1 Suppl 1): 81-7.

23. Hirawa $N$, Uehara $Y$, Kawabata $Y$, et al. Mechanistic analysis of renal protection by angiotensin converting enzyme inhibitor in Dahl salt-sensitive rats. J Hypertens 1994; 12: 909-18. 